\title{
Bradeion Project: Monitoring and Targeting of Cancer: Molecular Marker Diagnosis of Cancer by Fluorescence Correlation Spectroscopy (FCS)
}

\author{
Tomoo Tanaka ${ }^{1,5}$, Yoshiaki Kawamura ${ }^{1}$, Yukio Usui ${ }^{1}$, Toshiro Terachi ${ }^{1}$, Fumihiro Kimura ${ }^{2}$, \\ Tomohiko Asano ${ }^{2}$, Masamichi Hayakawa ${ }^{2}$, Norihiro Sakai ${ }^{3}$, Shinji Morimoto ${ }^{3}$, Yoshiyuki Mogi ${ }^{3}$, \\ Hideomi Fujiwara ${ }^{3}$, Nobuhiko Yamamoto ${ }^{4}$, Kiyoshi Komori ${ }^{5}$ and Manami Tanaka*,
}

\author{
${ }^{1}$ Tokai University School of Medicine, Shimokasuya 143, Isehara, Kanagawa 259-1193, Japan \\ ${ }^{2}$ Department of Urology, National Defense Medical College, Namiki 3-2, Tokorozawa, Saitama 359-8513, Japan \\ ${ }^{3}$ Tsuchiura Kyodo General Hospital, 11-7 Manabe Shin-Machi, Tsuchiura, Ibaraki 300-0053, Japan \\ ${ }^{4}$ Sinjuku Center Bldg. Clinic, Shinjuku Center Bldg. 5F, 1-25-1 Nishi-Shinjuku, Shinjuku, Tokyo 163-0605, Japan \\ ${ }^{5}$ Bradeion Project, National Institute of Advanced Industrial Science and Technology (AIST), Bldg. Tsukuba Central 6- \\ 1-421, Higashi, Tsukuba Science City, Ibaraki 305-8566, Japan
}

\begin{abstract}
The human Sept4/Bradeion GTPase (56Kda) is specifically expressed in colorectal cancer, malignant melanoma, prostate cancer and other urologic cancers such as renal cell carcinoma and bladder cancer. Significance of Bradeion is that, 1) 'all or none' expression, 2) no genetic alterations among patients, and 3) strong tissue-and cell typespecificity. This character satisfies the desired criteria as a target to monitor and control cancer. With the advent of current technology development in nano-scale, we have succeeded to develop the effective serum test for early diagnosis of cancer. Fluorescence correlation spectroscopy (FCS) can directly and quickly analyse protein-protein binding in a sample solution by reflecting the molecular weight of the labeled molecules. We present a novel and successful diagnostic methods by the quantitative detection of Sept 4 /Bradeion $\beta$ in the patients' serum using monoclonal antibodies. The resulting diagnostic efficacy was as follows; $100 \%$ (colorectal cancer), $89.0 \%$ (prostate cancer), $92.3 \%$ (renal cell carcinoma), and $89.0 \%$ (bladder cancer). More importantly, the increased Bradeion titers decreased to the normal level after one week (polypectomy with colonoscope) to 3 weeks after surgical detection of the cancer.
\end{abstract}

Keywords: Human Sept4 GTPase, Bradeion, colorectal cancer, prostate cancer, serum test, Fluorescence correlation spectroscopy (FCS).

\section{INTRODUCTION}

Molecular diagnostic method has long been under investigation, but not yet succeeded to provide a solution for its promise. With the advent of current technology development in nano-scale, many applications and hints are appearing every journal to provide a huge amount of new data. However, these devices have not met enough practical and effective contents with better cost-performance, which satisfies the requirement of medical doctors.

We have initiated molecular and biochemical approach to provide an effective marker to produce rationally designed molecular-targeted diagnostic tools [1-3]. Septins are GTPbinding proteins that assemble into homo- and heterooligomers and filaments [4-5]. Although little is known concerning the molecular functions of mammalian septins, we identified that human Sept4/ Bradeion $\beta$ has expression in limited human cancers (colorectal carcinoma, malignant melanoma, prostate cancer and other urologic cancers)

*Address correspondence to this author at the Bradeion Project, National Institute of Advanced Industrial Science and Technology (AIST), Bldg. Tsukuba Central 6-1-421, Higashi, Tsukuba Science City, Ibaraki 305-8566, Japan; Fax: +81-298-61-6046; E-mail: tanaka-manami@aist.go.jp presumably occurred as a result of carcinogenesis [1]. It is strongly limited in cancer cells irrespectively to other possible oncogene mutations. Bradeion seems to function as characteristic septin GTP-binging GTPase for accelerated cytokinesis and cell separation in a cancer cell- and contextspecific fashion $[1,6]$, and thus without any point mutations among individuals. Suppression of Bradeion gene expression by antisense ribozymes treatment resulted in G2 arrest and cell death ${ }^{3}$. Russell and Hall indicated that the available data suggest that at least some septins can be implicated in human (and murine) neoplasm [7]. In order to produce an effective diagnostic tool, we have already reported two independent approaches, one for antibody-based immunochromatographic rapid test (membrane strip), and another is quantitative RT-PCR [2].

Our aim and objectives should satisfy the requirements as listed below;

1) Patient serum as a sample, easy to obtain for laboratory screening on admission and also to be simultaneously used for the other clinical tests, 2) Simplicity of the technique and methods, 3) No false negative and low false positive results, 4) The best cost performance including maintenance of the devices, 5) Strong reproducibility and reliability, 6) Safety of the reagents and technique itself at 


\section{Antibody Labeling}

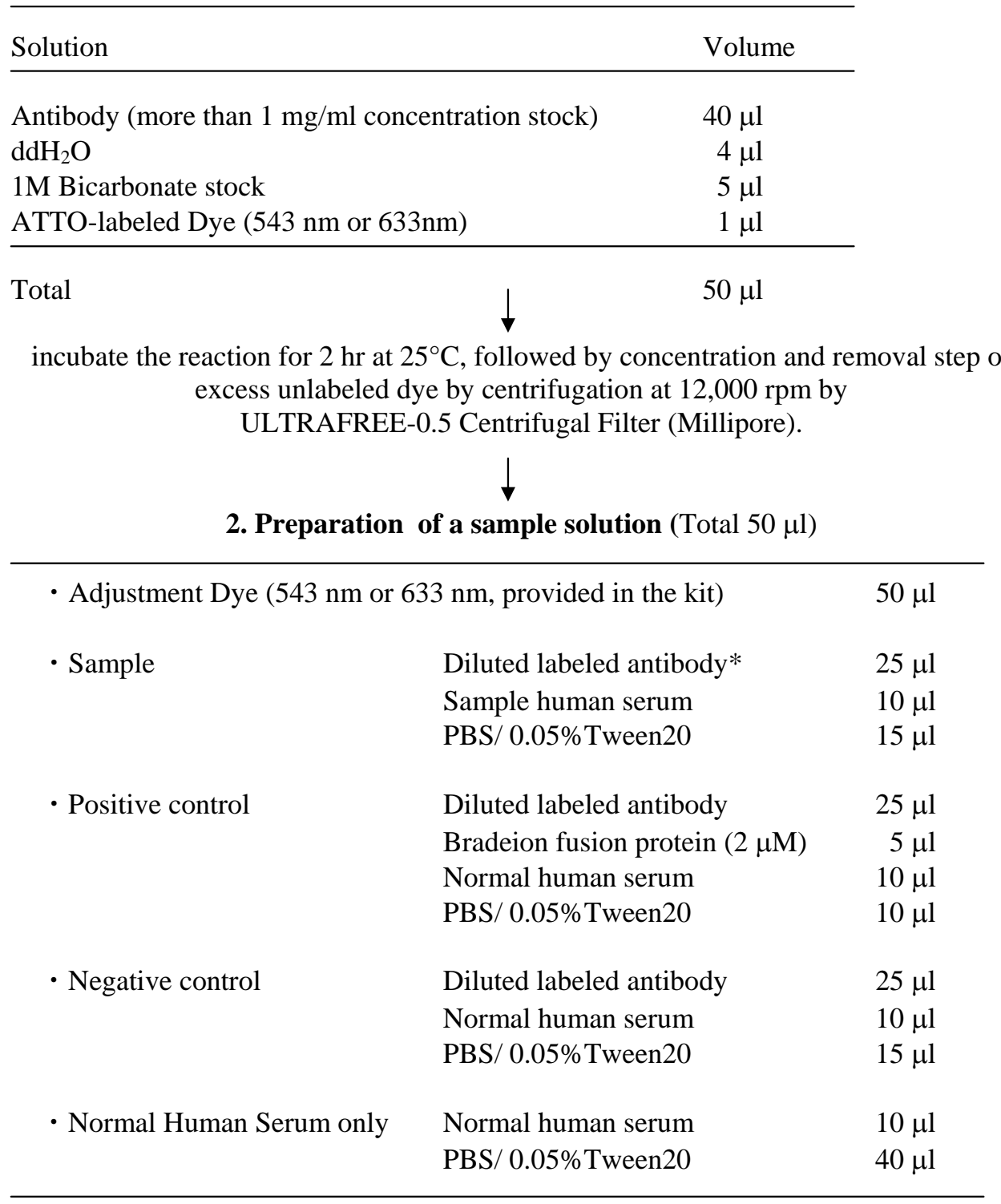

*The dilution should be performed to make the quantity of labeled IgG, ca. $2.0 \mathrm{ng} / \mu \mathrm{l}$, or particle number $=2.0$ by the MF20 software.

\section{FCS measurement (10 sec $x$ 3)

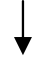

\section{Data analysis by MF20 software (for $\mathrm{K} 1$ diffusion time)}

Adjust the basal (level 0) line from the results of negative controls

Fig. (1). An outline of FCS 'mix and measure' method with a trouble-shooting guide.

use, at storage, and more importantly, at disposal. Based on the previous examinations using membrane immunochromatographic method and surface plasmon resonance (SPR) using BIACORE (GE Healthcare), we employed antigenantibody binding reaction to detect the presence of cancer marker molecule in the patient serum. Bradeion $\beta$ shows a merit to be expressed only in cancer cells, not in the normal tissue and serum.
Laser-induced fluorescence detection of single fluorescent molecules represents the nano-scale level of sensitivity for fluorescence-based assays in analytical chemistry, biology, and medicine [8]. The recent improvements in single-molecule detection using FCS technique lead us to expect diagnostic procedure, together with drug candidate validation using sequences and monoclonal antibodies against Bradeion $\beta$ (IBL, Gunma, 
Japan). The demerit of FCS chiefly lies in the molecule size to be measured. The resulting molecule to be measured should have a reasonable difference from the basal level (at least $50 \mathrm{KDa}$ in size). In the present study, the basal molecule is fluorescent-labeled monoclonal antibody (IgG, approximately $150 \mathrm{KDa}$ ), and the Bradeion-binding antibody will be at least $210 \mathrm{KDa}$. Since the Bradeion generally forms a dimmer in vivo [9-11], resulting size of the binding molecule should be $260 \mathrm{KDa}$, which will be enough to apply FCS technique (260 KDa vs. $150 \mathrm{KDa})$.

The present study introduces a novel and practical method for reliable clinical diagnosis of Sept4/ Bradeion $\beta$ expressed cancer, renal cell carcinoma, by 'mix and measure' protocol with high reproducibility and reasonable cost-performance (ranges 1 to 10 cents per sample).

\section{MATERIALS AND METHODS}

\section{Fluorescence Correlation Spectroscopy}

FCS measurements were performed using the MF20 molecular interaction analytical system (Olympus, Tokyo, Japan), which was developed in collaboration with Evotec BioSystems AG (Hamburg, Germany). It employs a singlemolecule fluorescence detection system and is equipped with various analytical methods including FCS. The consequent methodology was shown in Fig. (1). For the validation and trouble shooting, the following points should be carefully confirmed; 1) the purification of IgG by Protein $\mathrm{G}$ column such as HiTrap ${ }^{\mathrm{TM}}$ Protein G HP Column, followed by high concentration of the protein by PD-10 Desalting Columns (GE Healthcare) was essential. The concentration of starting antibody stock solution is desired to be more than $1 \mathrm{mg} / \mathrm{ml}$. The removal of sodium azide was performed by ULTRAFREE-0.5 Centrifugal Filter Device (Millipore), and followed by the labeling with ATTO-labeled Dye (ATTOTEC, Siegen, Germany); 2) Before fluorescent-labeling of purified antibody, it is very important to confirm the removal of the excessive dye remaining in the labeling mix to the negligible level. Use ULTRAFREE-0.5 Centrifugal Filter Device (Millipore) or equivalent equipments for this purpose. The labeling ratio should be carefully checked several times by FCS measurement in the process.

FCS exactly determines the diffusion time of labeled molecules passing by the laser spot, reflecting the molecular weight of the labeled molecules. The increased molecular weight by protein-protein binding can be observed by its slow diffusion time. Another merit of this analysis is that FCS can analyze the complex formation under the equilibrium condition in a solution mixture $[8,9]$.

The mixture was added to a 384-well Glass Bottom Microplate (Olympus, Tokyo, Japan), and the measurements were carried out in a sample volume of $50 \mu 1$. The optical system was also automatically adjusted for each measurement. For the detection of antibodies labeled with ATTO 543 or 633 (emission wavelength: $543 \mathrm{~nm}$ or $633 \mathrm{~nm}$; ATTO-TEC, Siegen, Germany), the He-Ne laser was used.

\section{Monoclonal Antibodies}

The Bradeion monoclonal antibodies hired in the present study has been IgG purified and concentrated by IBL (Immuno-Biological Laboratories, Co., Ltd.), Japan. Three different antibodies were used according to their sensitivity and selectivity. The highest sensitivity was observed with AT9A5, and the highest selectivity with AF6D6. MS1A9 stands middle in both characters [2].

\section{Human Serum Samples}

The serum samples were taken at the time of admission. The research ethics committee of Tokai University School of Medicine, National Defense Medical College, Tsuchiura Kyodo General Hospital, and National Institute of Advanced Industrial Science and Technology approved the study and all patients provided written informed consent. Total 2 - 10 $\mathrm{ml}$ serum from the patients and from the healthy volunteers were obtained and stored for the present study.

In addition to the age-matched normal serum from Japanese volunteers, commercially available normal human serum pool (Chemicon International, Co. Ltd.) was also used in each examination.

\section{RESULTS AND DISCUSSION}

\section{Standardization of FCS Measurement Protocol}

For standardization of the technique, we first used different concentrations of recombinant Bradeion solution $[1,2,6]$ ranging 0 to $200 \mathrm{vM}$, which were incubated with fluorescent-labeled anti-Bradeion monoclonal or polyclonal antibody with or without normal human serum $(20 \%)$. We tested series of monoclonal antibodies (11 against full-length fusion protein [2], 5 against partial amino acid sequences which represent enzymatic activity; IBL, Gunma, Japan) by single or mixture of 3 to 4 antibodies at a time. In the present study, monoclonal antibody AT9A5 [2] showed the best efficacy, and two other monoclonal antibodies also showed enough efficacy to be used (MS1A9, AF6D6, and all mixture) [2]. The diagnostic efficacy of each monoclonal antibody could be predicted using membrane immunochromatography (membrane test-strip), and by the surface plasmon resonance (BIACORE).

The optimized protocol was shown in Fig. (1). Calibration of the diffusion time was automatically performed by software attached to the MF20, and measured molecular weights of binding molecule can be calculated (Fig. 2a and 2b). Calibration of the diffusion time was automatically performed by software attached to the MF20, and measured molecular weights of binding molecule can be calculated according to the following equations;

$$
\begin{aligned}
& D T=\text { bottom }+(\text { top }- \text { bottom }) \times \\
& \frac{K d+[A]_{0}+[B]_{0}-\sqrt{\left(K d+[A]_{0}+[B]_{0}\right)^{2}-4[A]_{0}[B]_{0}}}{2[A]_{0}} \\
& {[B]_{0}=\frac{[A]_{0} Y^{2}-\left(K d+[A]_{0}\right) Y}{Y-1}} \\
& Y=\frac{D T-\text { bottom }}{\text { top-bottom }} \\
& \text { DT: } \quad \text { Diffusion time }
\end{aligned}
$$

Bottom: Diffusion time of normal control

Top: Maximum diffusion time 
$\mathbf{a}$

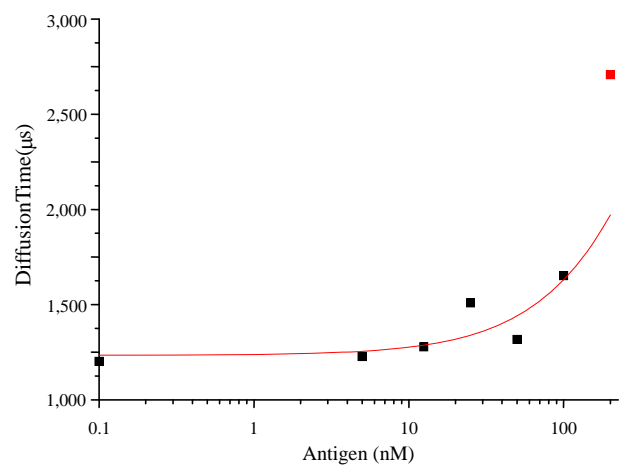

b

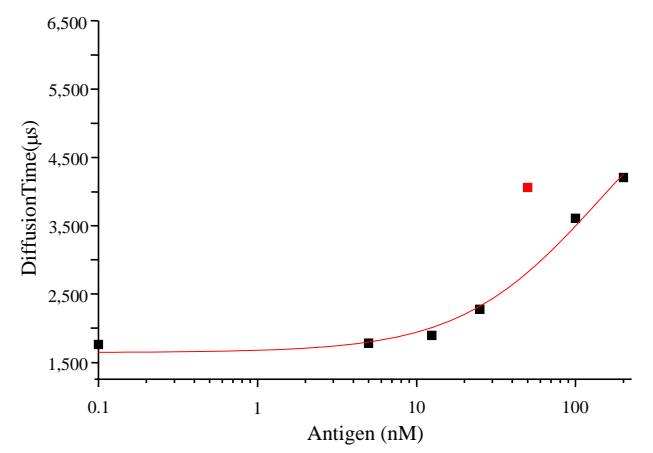

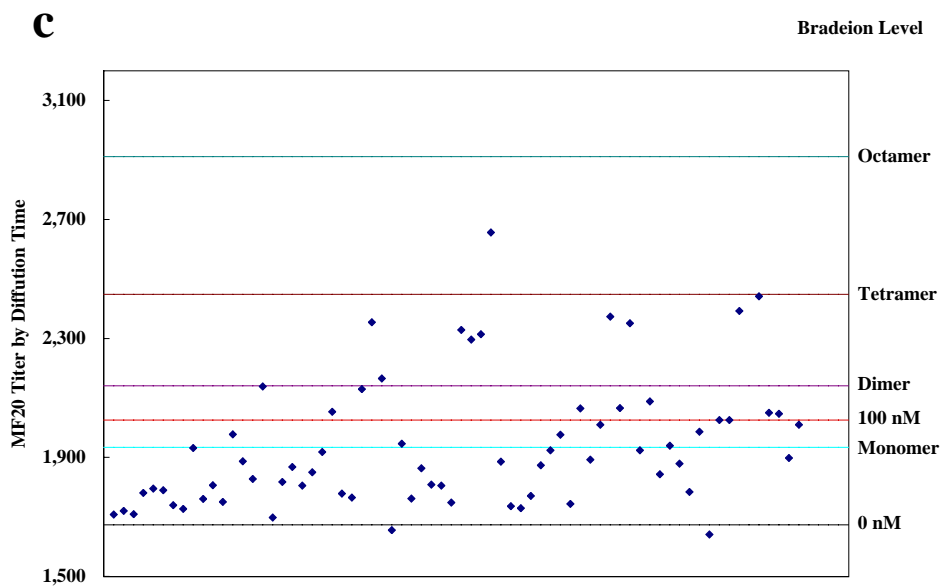

Fig. (2). Human Sept 4 /Bradeion $\beta$ detection by FCS $[8,9]$. (a) Standard curve of Sept 4 / Bradeion $\beta$ measurement by FCS (only mixture of fluorescent-labeled antibody and recombinant Bradeion without serum). The signals of each sample automatically detected for 20 sec 5 times. (b) Standard curve using samples with $20 \%$ normal serum (Chemicon International, Co., Ltd.). (c) FCS titers of the patients' serum of renal cell carcinoma using ATTO-labeled monoclonal antibody AT9A $5^{2}$. Titers shown in the left bar (vertical axis) is diffusion time, and the binding molecule size in the right bar (horizontal axis). The basal level 0 level titer was defined by measurement of $20 \%$ normal serum with fluorescent-labeled antibody.

$[\mathrm{A}]_{0}: \quad$ Concentration of fluorescent-labeled antibody $(2 \mathrm{nM})$

$[B]_{0}$ : Concentration of the binding molecule $(\mathrm{nM})$

Kd: Diffusion constant

We validated the conditions using the same sample for 3 sec 3 and 10 times; for $10 \sec 3,5$ and 10 times; and also for $20 \mathrm{sec} 3$ and 5 times, respectively. The mixture of the sample solution did not require any further incubation, and the measurement for $10 \mathrm{sec}$ three times were enough to obtain the data. Each dot represents a mean data of three independent measurements of patient serum samples for 10 sec three times.

Theoretically, diffusion time of only labeled-antibody (ca. $150 \mathrm{kDa}$ ) should be $600-620$ by $543 \mathrm{~nm}$, and 16001800 by $633 \mathrm{~nm}$ as indicated in the manufacturer's instructions, but the data varied among experiments in reality. In the present study, the basal level of diffusion time using $543 \mathrm{~nm}$ labeling resulted in a wide and instable range of lower titer (500-750), and a significant binding could not be observed, either. We thus performed the experiments by $633 \mathrm{~nm}$ labeling, and that with careful calibration of the standard bottom diffusion time at each time of the measurement.

We compared the results by incubation time at $4{ }^{\circ} \mathrm{C}$ up to overnight (16 hrs), $37{ }^{\circ} \mathrm{C}$ one to two hour, and simple mix at room temperature $\left(25{ }^{\circ} \mathrm{C}\right)$. Simple 'mix and measure' method at room temperature with no incubation time yielded at most practical and reproducible results. Unlike the other pathologic immunodetection methods such as immunoprecipitation, Western blot, and/or histological reactions, the longer incubation at any temperature did not show any enhancement or increase of binding activity at all. Salt concentration did not show a significant difference, and any commercially available $\mathrm{PBS}$ solution within range of $\mathrm{pH} 6.8$ 8.0 can be used.

The typical result with the protocol using 70 renal cell carcinoma (stages I to IV) was demonstrated in Fig. (2c). The measured molecular weights differs among patients, 
irrespectively to the stages of the cancer, which might be the results of conformation change of the molecule in the patient serum as suggested previously [7, 8]. This might be partly because the released protein from cancer cells would be metabolized to be the appropriate stable conformation, and also because of the other factors affecting to the binding motifs of the molecule. We tried to visualize the conformation using fusion protein by the atomic force microscopy (AFM) [12]. The Bradeion $\beta$ (26 kDa) was shown in Fig. (3). The previous reports proved that it usually appears as a dimmer [10,11].

Two samples showed negative results below the basal line, one of which was stage Ia carcinoma below the tumor size of $1.1 \mathrm{~mm}$, and the other was detected positive with the other monoclonal antibody (MS1A9 and mixed monoclonal antibodies). There was an advantage of double check measurement using the other monoclonal antibodies for those patients who could not rule out the possibility of cancer from the other clinical examinations. Polyclonal antibody was simultaneously used, but the sensitivity was significantly lower, just as the same in the other techniques reported [2].

Compared with the other diagnostic technology, we have previously reported that the membrane test-strip methods are very economical procedure with no false negative results ${ }^{2}$. However, the demerits are 1) not so quantitative as expected, and 2) with false positive ratio up to $37 \%$. The measurement using BIACORE was so far effective to choose the effective antibodies, however, it costs too high (up to $\$ 20.00$ ) for measurement and analysis with much longer duration, with much less sensitivity compared with FCS (10\% or less). From these evaluation and validation, we only used the SPR technology for the selection of antibody to show high value of association constant to antigen for further experiments. It should be emphasized that there are few antibodies which can be used for the immunological detection of cancer marker, and that the association constant should be first tested and proven by the other devices. In addition, many devices failed to be applied in clinical use simply because of the lack of appropriate antibodies.
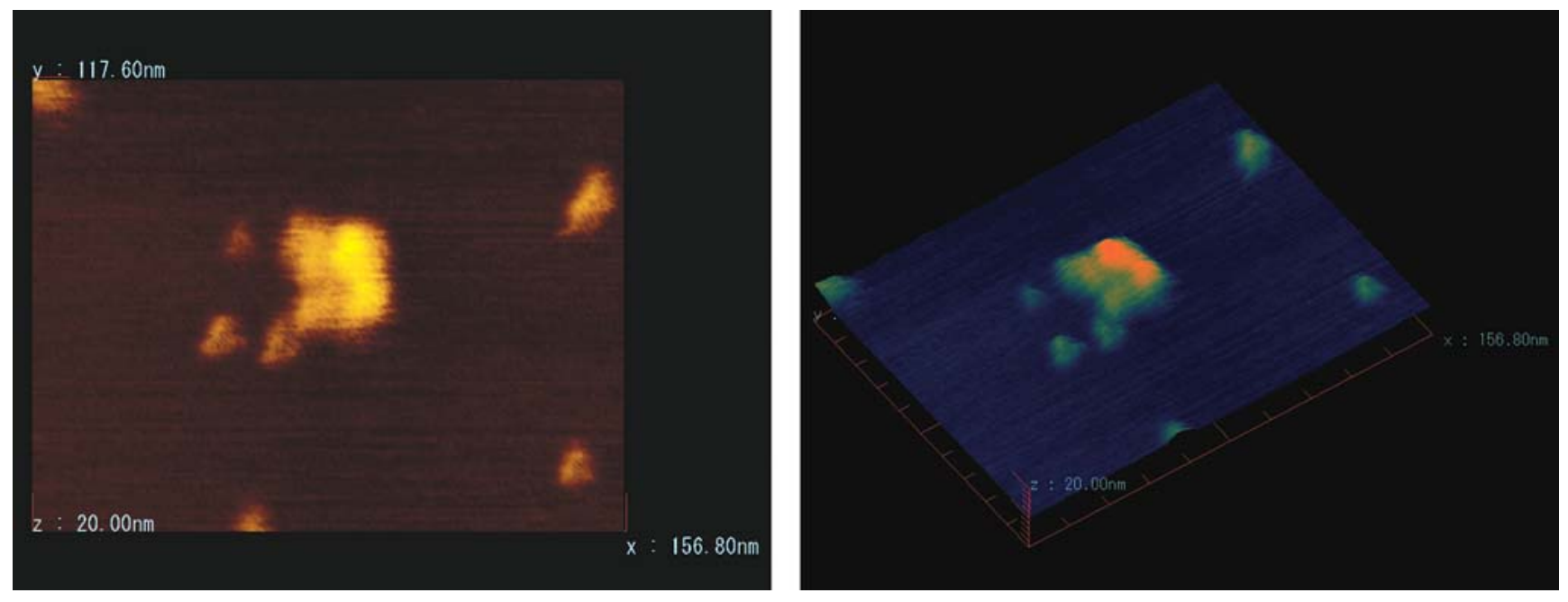

Efficacy of Bradeion Serum Test in Hospitalized Patients and Cancer-Positive Ratio in Healthy Population in Japan

Following to the successful development of serum test by FCS, we have started the stage of large-scale clinical trial. The results using total 124 serum samples from healthy volunteers and 377 cancer patients were shown in the present study.

As shown in Fig. (4a), $30 \%$ of healthy population with no clinical symptoms or complaints resulted in positive. Those positive cases were all negative in fecal occult blood test, and the other cancer markers such as $\alpha$-Fetoprotein, CA72-4, and carcinoembryonic antigen (CEA). The results of colonoscopy revealed one to multiple polyps in 22 cases (hyperplasia to late adenoma) in colon (Fig. 4b). All polyps were treated by finding (polypectomy) and designated to pathological analysis. Bradeion titers decreased to normal level 0 after one week after polypectomy, which clearly indicates the high Bradeion level was caused by the colon lesion.

The positive cases over 60 years old male, with elevated PSA titers, are currently monitored once in 3-6 months for any changes of the titer. In Japan, aggressive examinations such as digital rectal examination (DRE) was not specifically recommended, since it was widely known that over $50 \%$ male population has cancer in situ in prostate. However, two out of 14 Bradeion-negative persons spontaneously had DRE (18x18 total), which resulted in negative.

\section{Efficacy of Bradeion Serum Test in Hospitalized Patients}

The results of Bradeion serum test, currently analysed 377 cancer patients were shown in Fig. (5). Those patients have been hospitalized and treated chiefly by surgical operation.

In conclusion, we have demonstrated the current advancement of molecular diagnosis by FCS. The present study is the first to describe the effective application of the FCS devices, since there are yet very few monoclonal and/or polyclonal antibody which can be successfully and

Fig. (3). AFM image of the molecule as two dimers on a mica surface [10]. The detailed methodology was described in ref. [10] (see supplementary video). 
(a)

\begin{tabular}{|c|c|c|c|c|c|c|c|c|c|}
\hline \multirow[b]{3}{*}{ Age } & \multicolumn{3}{|c|}{ Male } & \multicolumn{3}{|c|}{ Female } & \multicolumn{3}{|c|}{ Total Number } \\
\hline & \multicolumn{2}{|c|}{ Positive } & \multirow{2}{*}{$\begin{array}{r}\text { No. } \\
103\end{array}$} & \multicolumn{2}{|c|}{ Positive } & \multirow{2}{*}{$\begin{array}{l}\text { No. } \\
17\end{array}$} & \multirow{2}{*}{$\begin{array}{c}\text { Positive } \\
33 \text { / }\end{array}$} & \multirow{2}{*}{$\begin{array}{r}\text { No. } \\
\mathbf{1 2 4}\end{array}$} & \multirow{2}{*}{$\begin{array}{c}\text { Pos. ratio (\%) } \\
26.6\end{array}$} \\
\hline & 31 & I & & 2 & I & & & & \\
\hline 20 ’s & 0 & & 10 & 0 & & 10 & 0 & 20 & 0.0 \\
\hline 30 ’s & 8 & & 22 & 0 & & 0 & 8 & 22 & 36.4 \\
\hline 40 's & 7 & & 24 & 1 & & 3 & 8 & 27 & 29.6 \\
\hline 50 's & 9 & & 29 & 1 & & 5 & 10 & 34 & 29.4 \\
\hline 60 's & 7 & & 21 & 0 & & 0 & 7 & 21 & 33.3 \\
\hline
\end{tabular}

\section{b}

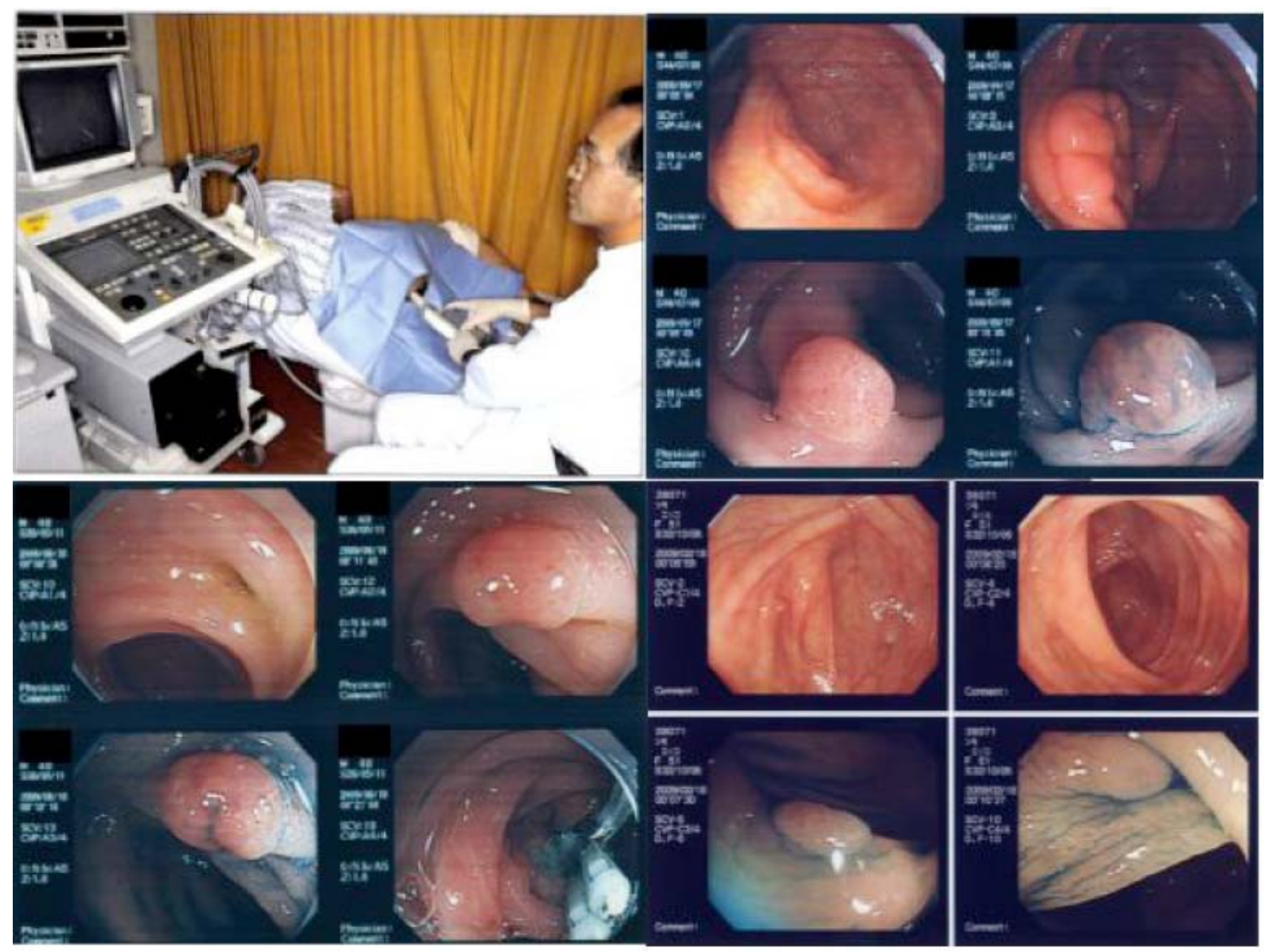

Fig. (4). Efficacy and cancer-positive ratio by Bradeion serum test. (a) Results in healthy population, All the examined individuals are healthy Japanese with no symptoms, no complaints, either no clinical disorders, (b) Lesions and polyps found in Bradeion positive cases.

practically used. The cost performance can become within 1 cent per one sample measurement, which depends on how many samples were analysed at a time. After successful labeling, $40 \mathrm{ng}$ (15 to $60 \mathrm{ng}$ range) of labeled-monoclonal antibody could be used for 1,000 samples (more than enough for two 384-well titer plates). The stock concentration of labeled IgG about $2.0 \mathrm{ng} / \mu \mathrm{l}$. The stock solution can be stored at most one month at $4{ }^{\circ} \mathrm{C}$ in dark. Moreover, the data can be reproduced after 16 hours when the sample solution is kept in dark at room temperature. The device requires only electricity and distilled water, and simple 'mix and measure' method with automated devices is very safe at use and also 


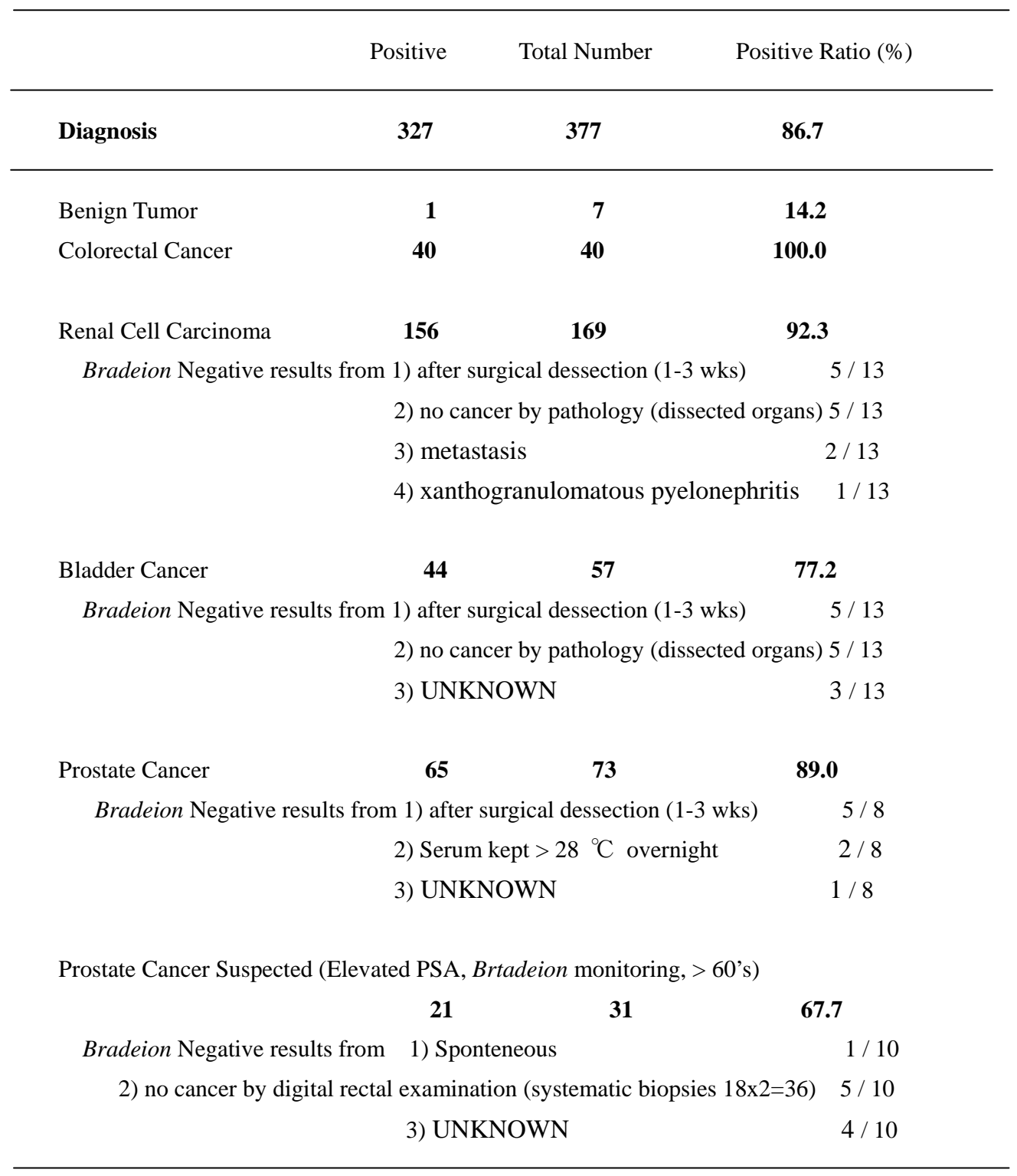

Fig. (5). Efficacy of Bradeion serum test in hospitalized patients.

at disposal. The devices and the software attached can be more simplified only for this purpose. The automated serum test thus drastically improves the time- and cost-consuming process for Sept 4 expressing cancers.

In addition, diagnostic efficacy has been continuously enhanced by the multiple marker measurements with the same protocol, such as 1) Anti-PSA (prostate specific antigen) mouse monoclonal antibody (Acris Antibodies $\mathrm{GmbH}$, Germany) for prostate cancer, 2) Anti-SCD mouse monoclonal antibody (CD.E10) (Anti-Acyl-CoA desaturase, Pierce Biotechnology, USA) for the detection of cancers without Bradeion expression, and 3) Anti-TGF $\beta 1$ (BIGH3) mouse-monoclonal antibodies (R \& D Systems, Inc., USA) for the detection of clear cell renal cell carcinoma [13].

\section{ACKNOWLEDGEMENTS}

We wish to thank Former President Akio Suzuki, President Iwao Ohyama, Prof. Dr. Fujie Numano, Tokyo Medical and Dental University, for the critical advice on clinical trial of the serum test. We also thank members of MEMS Department of OLYMPUS Corporation for helpful discussion and technical advice; Prof. Dr. Richard Charles Garratt, University of São Paulo, Brazil, for molecular structural analysis; and technical assistance to Mr. Nobuhiko Shirato, Tokyo Medical and Dental University and Ms. Yoko Tomiyama at AIST. We wish to thank many healthy volunteers, and patients kindly collaborated with the project.

\section{REFERENCES}

[1] Tanaka, M.; Tanaka, T.; Itoh, J.; Matsuda, T.; Hori, S.; Kijima, H. Characterization of tissue- and cell type-specific expression of a novel human septin family gene. Bradeion. Biochem. Biophys. Res. Commun., 2001, 286, 547-553.

[2] Tanaka, M.; Tanaka, T.; Kijima, H.; Itoh, J.; Matsuda, T.; Komori, K.; Seto, Y.; Misse, S.; Matsuzaki, S.; Hashimoto, Y.; Toma, H. Rapid and quantitative detection of a mammalian septin Bradeion as a practical diagnostic method of colorectal and urologic cancers. Med. Sci. Monit., 2003, 9, 61-68.

[3] Tanaka, M.; Kijima, H.; Itoh, J.; Matsuda, T.; Tanaka, T. Impaired expression of a human septin family gene Bradeion inhibits the 
growth and tumorigenesis of colorectal cancer in vitro and in vivo. Cancer Gene Ther., 2002, 9, 483-488.

[4] Macara, I.G.; Baldarelli, R.; Field C.M.; Glotzer, M.; Hayashi, Y.; Hsu, S.C.; Kennedy, M.B.; Kinoshita, M.; Longtine, M.; Low, C.; Maltais, L.J.; McKenzie, L.; Mitchison, T.J.; Nishikawa, T.; Noda, M.; Petty, E.M.; Peifer, M.; Pringle, J.R.; Robinson, P.J.; Roth, D.; Russell, S.E.; Stuhlmann, H.; Tanaka, M.; Tanaka, T.; Trimble, W.S.; Ware, J.; Zeleznik-Le, N.J.; Zieger, B. Mammalian septins nomenclature. Mol. Biol. Cell, 2002, 13, 4111-4113.

[5] Sirajuddin, M.; Farkasovsky, M.; Hauer, F.; Kühlmann, D.; Macara, I.G.; Weyand, M.; Stark, H.; Wittinghofer, A. Structural insight into filament formation by mammalian septins. Nature 2007, 449, 311-315.

[6] Russell, S.E.H.; Hall, P.A. Do septins have a role in cancer? Br. J. Cancer 2005, 93, 499-503.

[7] Eggeling, C.; Fries, J.R.; Brand, L.; Günther, R.; Seidel, C.A. Monitoring conformational dynamics of a single molecule by selective fluorescence spectroscopy. Proc. Natl Acad. Sci. USA, 1998, 95, 1556-1561.

[8] Jäger, S.; Brand, L.; Eggeling, C. New fluorescence techniques for high-throughput drug discovery. Curr. Pharm. Biotechnol., 2003, 4, 463-476.
[9] Garcia, W.; de Araújo A.P.U.; Lara, F.; Foguel, D.; Tanaka, M.; Tanaka, T.; Garratt, R.C. An intermediate structure in the thermal unfolding of the GTPase domain of human septin 4 (SEPT4/Bradeion- $\beta$ ) forms amyloid-like filaments in vitro. Biochemistry 2007, 46, 11101-11109.

[10] Garcia, W.; de Araújo, A.P.U.; de Oliveira Neto, M, O.; Ballestero, M.R.M.; Polikarpov, I.; Tanaka, M.; Tanaka, T.; Garratt R.C. Dissection of a human septin: definition and characterization of distinct domains within human SEPT4. Biochemistry 2006, 45 , 13918-13931.

[11] Hillebrand, S.; Carcia, W.; Cantú, M.D.; de Araújo, A.P.U.; Tanaka, M.; Tanaka, T.; Garrat, R. C.; Carrilho, E. Capillary electrophoresis-based method for in vitro monitoring of GTPase/ATPase activity and enzyme kinetics studies. Anal. Bioanal. Chem., 2005, 83, 92-97.

[12] Yokokawa, M.; Wada, C.; Ando, T.; Sakai, N.; Yagi, A.; Yoshimura, S.H.; Takeyasu, K. Fast-scanning atomic force microscopy reveals the ATP/ADP-dependent conformational changes of GroEL. EMBO J., 2006, 25, 4567-4576.

[13] Yamanaka, M.; Kimura, F.; Kagata, Y.; Kondoh, N.; Asano, T.; Yamamoto, M.; Hayakawa, M. BIGH3 is overexpressed in clear cell renal cell carcinoma. Oncol Rep., 2008, 19, 865-874.

(c) Tanaka et al.; Licensee Bentham Open.

This is an open access article licensed under the terms of the Creative Commons Attribution Non-Commercial License (http://creativecommons.org/licenses/by-nc/3.0/) which permits unrestricted, non-commercial use, distribution and reproduction in any medium, provided the work is properly cited. 